\title{
Comment on: "Team approach: Management of osteonecrosis in children with acute lymphoblastic" leukemia". An ounce of prevention is worth a pound of cure. Consideration of prophylactic pamidronate.
}

\author{
Bruce Bostrom ${ }^{1}$ \\ ${ }^{1}$ Children's Hospitals and Clinics of Minnesota
}

September 11, 2020

Jones et al. have given us an excellent guide for the management of symptomatic osteonecrosis in children and young adults with ALL. (1) I would like to present our 10 year retrospective concurrent control evaluation of pamidronate for reducing the incidence of symptomatic osteonecrosis which may be of interest.

Patients aged 10-28 years at time of ALL diagnosis were given intravenous pamidronate $(1 \mathrm{mg} / \mathrm{kg}$ IV over 2 hours) monthly for one year at the discretion of the primary oncologist, starting as early as possible after diagnosis.

Concurrent controls age 10-28 did not receive pamidronate. All were treated according to the concurrent COG protocols with intermittent dexamethasone during delayed intensification.(2) Imaging was performed if osteonecrosis was suspected based on symptoms. Patients with BCR-ABL ALL were excluded, as dasatinib may increase the risk of osteonecrosis. $(3,4)$

Patients were diagnosed between January 2010 and March 2018. They were censored at relapse (n=4; 2 controls) bone marrow transplant $(n=4 ; 3$ controls) or at last follow up. Data was analyzed 6/1/2020. The median follow up is 3.3 years from diagnosis to event or censoring. This retrospective study was approved by Children's Minnesota IRB. Data was entered into excel and transferred to SPSS version 23 for analysis.

There were 65 patients, 38 males, 27 females, of which 49 had B-lineage and 16 T-lineage ALL. Pamidronate was started during induction in $63 \%$ of patients, and before delayed intensification in $85 \%$. The mean, median and interquartile range for the number of pamidronate doses was 11.6, 12, 10.8 to 12. Pamidronate was used in 26 patients, with four subsequently developing symptomatic osteonecrosis. There were 39 concurrent controls who did not receive pamidronate with 14 developing osteonecrosis. Five from this group have since received joint replacements. There were no short or long term side-effects from pamidronate infusions including osteonecrosis of the jaw or hypocalcemia.

The incidence of symptomatic osteonecrosis by Kaplan-Meier analysis with was $16 \%$ with pamidronate vs. $39 \%$ in controls (figure 1). P-value is significant at 0.043 (Breslow Generalized Wilcoxan). There was no significant difference in the leukemia lineage, gender distribution or Body Mass Index (BMI) at diagnosis between groups. For all patients the mean, median, and interquartile range for BMI was 25.8; 22.0; 14.2 to $28.8 \mathrm{Kg} / \mathrm{m}^{2}$.

The age at diagnosis was significantly greater in the pamidronate group with a mean, median and Interquartile range $18.4 ; 18.6 ; 13.8$ to 23.4 years for pamidronate patients vs. $15.6 ; 15.7 ; 11.5$ to 19.9 in concurrent controls (independent means t-test $\mathrm{p}=0.01$ ). Age was not significant for osteonecrosis in Cox Proportional Hazard analysis $(\mathrm{p}=0.10)$. 
Study limitations include small numbers of patients from a single institution and lack of a randomized control group. Strengths of the study are the long duration of followup, as most of the patients are beyond the peak risk time for osteonecrosis. We hope these results even with its limitations would spark interest in a randomized trial of pamidronate in patients at high risk of symptomatic osteonecrosis.

Author Contributions and Disclosures: Bruce Bostrom was the sole contributor to this submission and has no conflict-of-interest to disclose. Jack Knutson assisted with data collection as part of a high school senior mentor connection project. Char Bostrom provided invaluable editorial assistance.

Figure 1 legend : Incidence of symptomatic osteonecrosis from time of ALL diagnosis in patients who received prophylactic pamidronate and concurrent controls.

\section{References}

1. Jones LC, Kaste SC, Karol SE, et al. Team approach: Management of osteonecrosis in children with acute lymphoblastic leukemia [published online ahead of print, 2020 Aug 29]. Pediatr Blood Cancer. 2020;e28509. doi:10.1002/pbc.28509

2. Mattano LA Jr, Devidas M, Nachman JB, et al. Effect of alternate-week versus continuous dexamethasone scheduling on the risk of osteonecrosis in paediatric patients with acute lymphoblastic leukaemia: results from the CCG-1961 randomised cohort trial. Lancet Oncol. 2012;13(9):906-915. doi:10.1016/S1470-2045(12)70274-7

3. Won AM, Boddu P, Otun AO, Aponte-Wesson R, Chambers M. Chronic myelogenous leukemia presenting with osteonecrosis of the jaw as a rare but debilitating toxicity of dasatinib: a case report and literature review. Oral Surg Oral Med Oral Pathol Oral Radiol. 2018;126(4):e208-e211. doi:10.1016/j.oooo.2018.05.008

4. Yassin MA, Moustafa AH, Nashwan AJ, et al. Dasatinib Induced Avascular Necrosis of Femoral Head in Adult Patient with Chronic Myeloid Leukemia. Clin Med Insights Blood Disord. 2015;8:19-23. Published 2015 Jul 23. doi:10.4137/CMBD.S24628

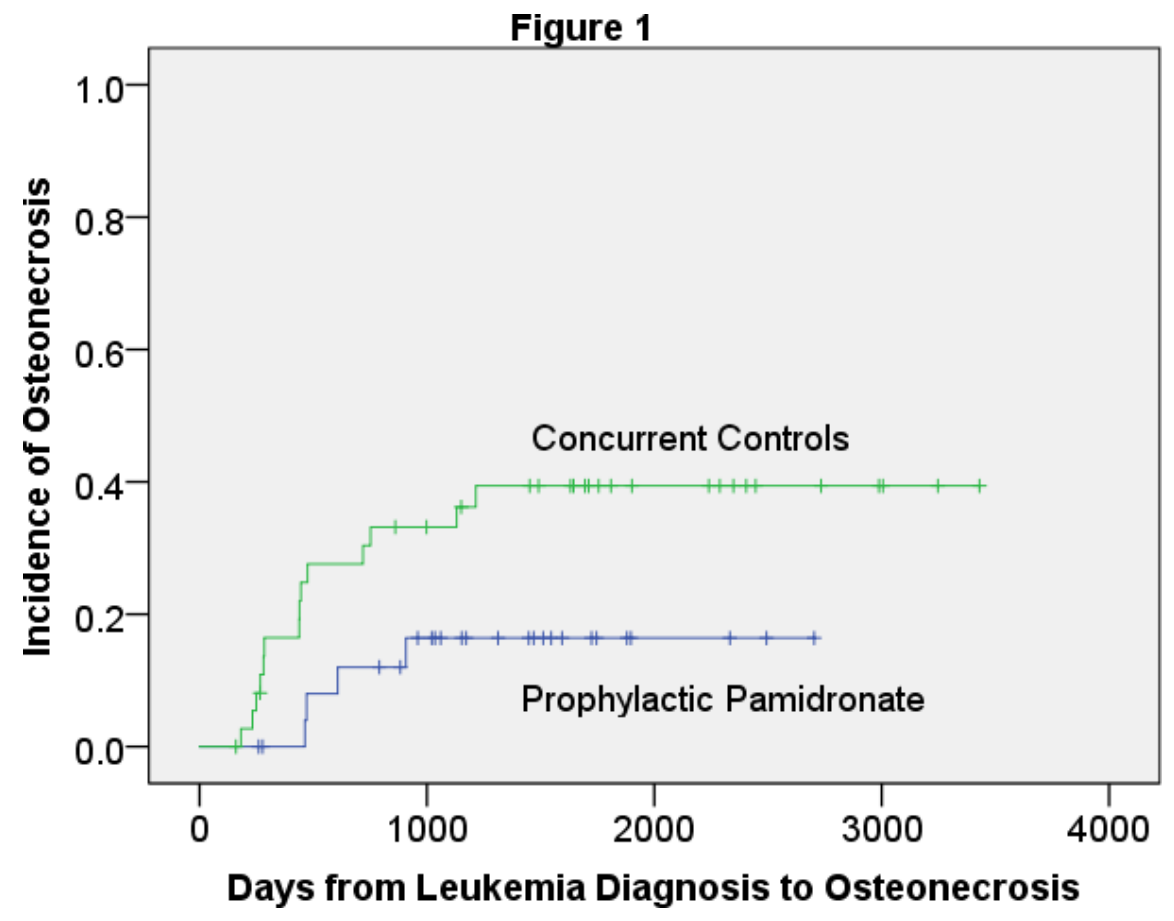

